\title{
Non-cirrhotic portal hypertension in an ankylosing spondylitis patient
}

\author{
Sukki Park, Ji Hyun Lee, Joon Sul Choi, Hyun Woo Kim, Beom Jin Shim, \\ Won Kyu Choi, Sang Hyun Kim \\ Division of Rheumatology, Department of Internal Medicine, Maryknoll Medical Center, Busan, Korea
}

\begin{abstract}
Idiopathic non-cirrhotic portal hypertension (INCPH) is a disease with an uncertain etiology consisting of non-cirrhotic portal hypertension and portal pressure increase in the absence of liver cirrhosis. In INCPH, patients exhibit normal liver functions and structures. The factors associated with INCPH include the following: Umbilical/portal pyremia, bacterial diseases, prothrombic states, chronic exposure to arsenic, vinyl chloride monomers, genetic disorders, and autoimmune diseases. Approximately $70 \%$ of patients present a history of major variceal bleeding, and treatment relies on the prevention of complications related to portal hypertension. Autoimmune disorders associated with INCPH are mainly systemic sclerosis, systemic lupus erythematosus and rheumatoid arthritis. To the best of our knowledge, a case of ankylosing spondylitis (AS) associated with INCPH has not been reported thus far. Therfore, we report our experience of a patient with AS accompanied by INCPH, who showed perisplenic varices with patent spleno-portal axis and hepatic veins along with no evidence of cirrhosis on liver biopsy, and provide a brief literature review.
\end{abstract}

Keywords: Portal hypertension; Ankylosing spondylitis; Perisplenic varix

\section{INTRODUCTION}

Idiopathic non-cirrhotic portal hypertension (INCPH) is a rare condition characterized by increased portal pressure with patent portal and hepatic veins in the absence of cirrhosis. The underlying pathophysiologic mechanisms of INCPH still remain unclear. INCPH has been associated with various conditions, including immunologic disorders, recurrent infections, medications and toxins, genetic disorders, and prothrombic conditions [1]. Histological features involve a wide range of non-specific changes, including sinusoidal dilatation, phlebosclerosis, portal fibrosis, and nodular regenerative hyperplasia

Received: July 8, 2016, Revised: October 29, 2016

Accepted: November 17, 2016

Corresponding Author: Ji Hyun Lee, Division of Rheumatology, Department of Internal Medicine, Maryknoll Medical Center, 121, Junggu-ro, Jung-gu, Busan 48972, Korea

Tel: +82-51-461-2732, Fax: +82-51-441-6950

E-mail: eyerheu1@nate.com
[2]. Moreover, INCPH is a diagnosis of exclusion, and the diagnosis is made when the following criteria are fulfilled: (1) evidence of portal hypertension, (2) doppler ultrasound showing patent portal and hepatic veins, (3) liver biopsy showing no cirrhosis, (4) exclusion of conditions that cause cirrhosis, such as chronic viral hepatitis, alcoholic liver disease, nonalcoholic steatohepatitis, obesity, or autoimmune hepatitis, and (5) exclusion of chronic vitamin A intake, exposure to copper sulphate, vinyl chloride monomer, or past angiography with thrombin sulphate [3]. Treatment is aimed to prevent potential complications of portal hypertension, and unfortunately, there is no available treatment to date that modifies the course of the disease or prevents complications.

We experienced a rare case of INCPH in a patient with ankylosing spondylitis (AS). INCPH is associated with immunological disorders, but it is mainly reported in patients with systemic sclerosis (SS), systemic lupus erythematosus (SLE), autoimmune hepatitis, and rheumatoid arthritis (RA) $[1,4,5]$. To date, INCPH in a patient with AS has not been reported.

Copyright (C) 2018 Yeungnam University College of Medicine

This is an Open Access article distributed under the terms of the Creative Commons Attribution Non-Commercial License (http://creativecommons.org/licenses/by-nc/4.0/) which permits unrestricted non-commercial use, distribution, and reproduction in any medium, provided the original work is properly cited. 
Therefore, we report INCPH in a patient with AS along with a brief literature review.

\section{CASE}

An 82-year-old female patient presented lower back pain that has been aggravated for the last 1 month. She was initially diagnosed with AS 16 years ago at a local clinic. She had been taking acetaminophen $325 \mathrm{mg}$, tramadol $37.5 \mathrm{mg}$, and aceclofenac $100 \mathrm{mg}$, twice a day. She underwent both total hip re- placement due to degenerative changes. She denied using alcohol and tobacco as well as taking any vitamins or other dietary supplements. She was not considered obese (body mass index $22.6 \mathrm{~kg} / \mathrm{m}^{2}$ ). The modified Schobers test was positive $(3.5 \mathrm{~cm})$; the fingertip to ground distance was $7 \mathrm{~cm}$; and chest expansion measured $3.5 \mathrm{~cm}$, which is considered to be outside the normal range. There was no flexion deformity of the cervical spine. Additionally, there was no tenderness at the sacroiliac joint on physical examination, and she showed no signs of arthritis or enthesitis.
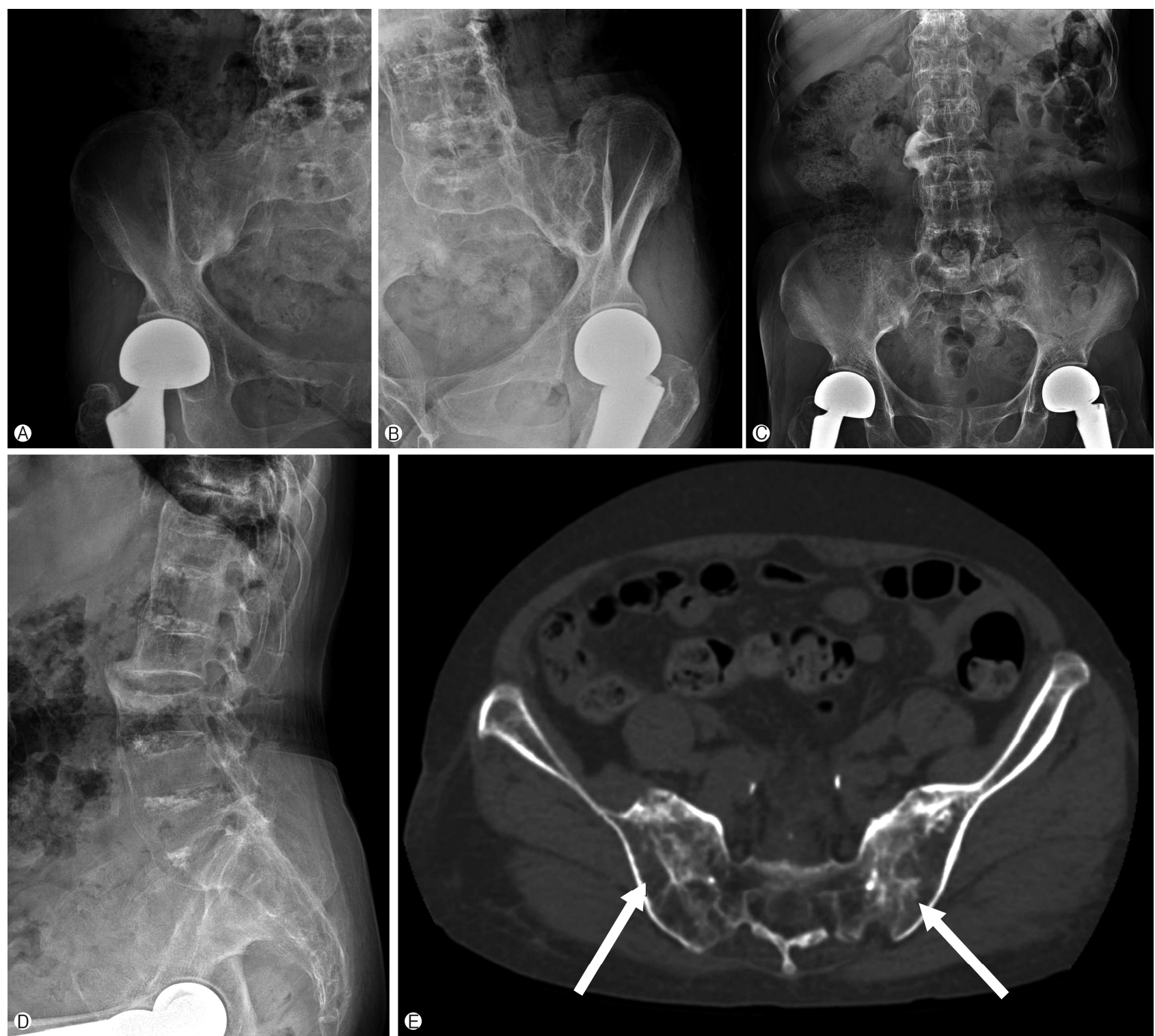

Fig. 1. Simple X-ray of sacroiliac joint, right (A), left (B), anteroposterior (C), and lateral radiographs (D) of the lumbosacral spine showed bilateral sacroiliitis and syndesmophytes of lumbar spines. Computed tomography image of the sacroiliac joints (E) showed erosions and eburnation (arrows). 
On laboratory findings, the erythrocyte sedimentation rate was $47 \mathrm{~mm} / \mathrm{hr}$ (range, $0-30 \mathrm{~mm} / \mathrm{hr}$ ) and high-sensitivity C-reactive protein was $18.22 \mathrm{mg} / \mathrm{L}$ (range, $0-0.5 \mathrm{mg} / \mathrm{L}$ ). Blood cell count was normal with platelet counts of $282,000 / \mu \mathrm{L}$. Human leukocyte antigen-B27, antinuclear antibody, and antiphopholipid antibodies were negative. Furthermore, rheumatoid factor was $6.8 \mathrm{IU} / \mathrm{mL}$ (range, $0-15 \mathrm{IU} / \mathrm{mL}$ ). Liver function tests and PT/INR were normal. HBsAg, antiHBs, and antiHCV were negative. There was no evidence of schistosomiasis on the serologic test.

On radiological findings, the sacroiliac joint on lumbar Xray and computed tomography (CT) showed bilateral sacroiliitis (grade 4) and bamboo spine consistent with AS (Fig. 1).

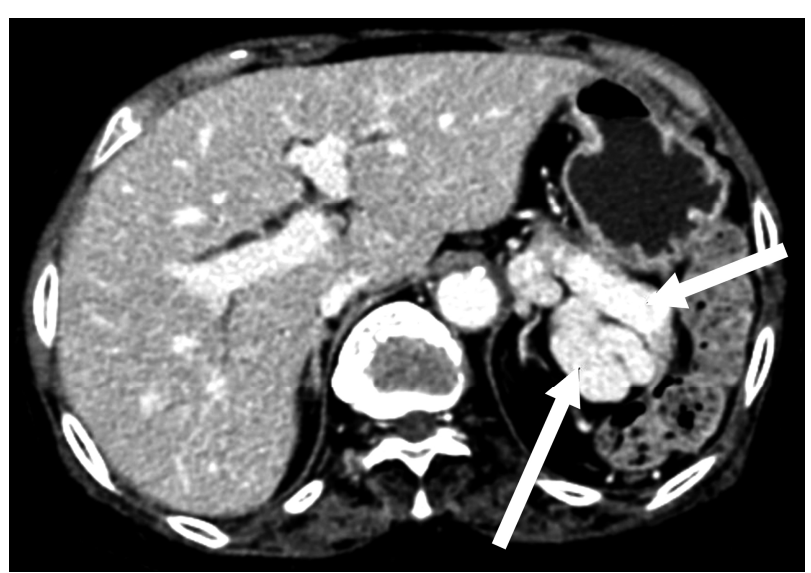

Fig. 2. Abdominal computed tomography shows perisplenic varices (arrows).

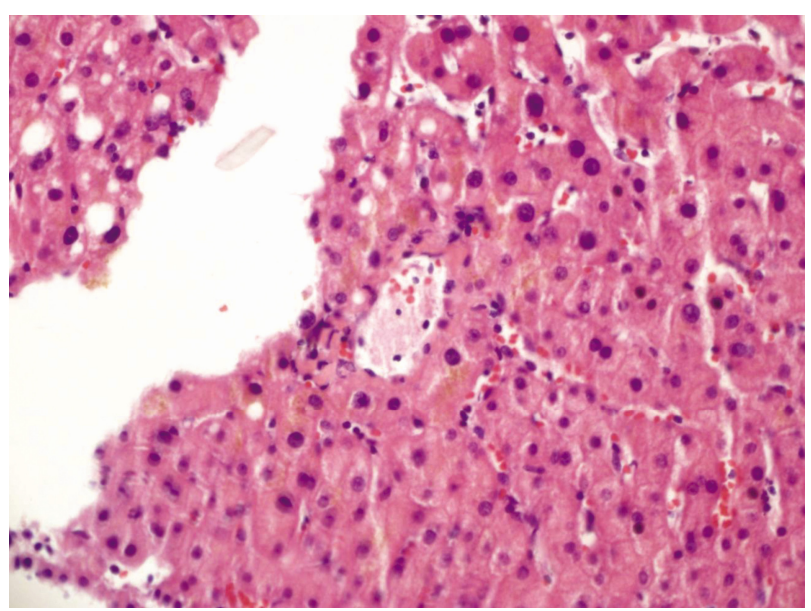

Fig. 3. Histologic finding shows mild and focal chronic hepatitis and cholestasis without any features suggesting fibrosis or cirrhosis $(H \& E$ stain, $\times 200)$.
Perisplenic varices and chronic obstructive nephropathy were observed on the abdominal CT (Fig. 2). Splenomegaly was not found. Doppler ultrasound showed patent portal and hepatic veins with no evidence of portal or splenic vein thrombosis.

We discovered gastric ulcer on upper gastrointestinal endoscopy without signs of bleeding. Moreover, there was no evidence of gastric and esophageal varices and portal hypertensive gastropathy. Liver biopsy showed mild and focal chronic hepatitis and cholestasis without any features suggesting fibrosis or cirrhosis (Fig. 3). A diagnosis of INCPH combined with AS was made. Methotrexate and celecoxib were prescribed and our patient's condition has been stable and uneventful, so far.

\section{DISCUSSION}

INCPH, also known as an idiopathic portal hypertension or non-cirrhotic portal fibrosis is a clinical condition secondary to increased pressure in portal vein in the absence of a known cause of liver disease or portal vein thrombosis (PVT) [1].

Although liver cirrhosis (LC) is the most frequent cause of portal hypertension, it can also develop in the absence of cirrhosis from a variety of disorders. Schistosomiasis is the most common cause. In addition, chronic liver diseases, such as non-alcoholic steatohepatitis, primary biliary cirrhosis, primary sclerosing cholangitis, congenital hepatic fibrosis, extrahepatic PVT, and Budd-Chiari syndrome, are the most common causes of non-cirrhotic portal hypertension. If all these aforementioned conditions have been rouled out, a diagnosis of INCPH can be made [6].

Both intrahepatic vascular obstruction and increased splanchnic blood flow have been suggested as responsible factors in determining portal hypertension in INCPH. An increased resistance is likely to result from the obstructed intrahepatic vessels and distorted intrahepatic angioarchitecture. Several hypotheses for the mechanisms responsible for the obliteration of portal venules have been proposed, including aberrant coagulation activation or thrombosis, acquired or inherited disorders of vascular remodeling, and endothelial injury from the immune cells [7]. An overproduction of nitric oxide, which is liberated in the sinus lining spleen cells, has been designated to be responsible for the dilatation of splenic sinuses. In patients with a more advanced disease, it is possible for increased 
intrahepatic resistance, which results from the obliteration of portal venous microcirculation, to lead to a further elevation of portal hypertension. It has also been speculated that increased production of endothelin-1 is likely to increase vascular resistance and stimulate periportal collagen production [6].

The etiology of INCPH includes the following: Chronic infections, exposure to medication or toxins, genetic disorders, and immunologic disorders. INCPH is frequently associated with immunological disorders, including SS, RA, and SLE. In patients with SS, a fibrogenetic process has been suggested to be an etiological factor. In patients with SLE, immunoglobulin interference with prostaglandin formation has been discovered to increase microthrombosis vulnerability. Another immunological disorder associated with INCPH is the anticardiolipin (aCL) antibody, in which its elevation can lead to the obliteration of small vessels. Primary hypogammaglobulinemia is also found in 70\% of INCPH patients. Although INCPH associated with AS has not been reported to date, the relationship between INCPH and AS could be considered in that the presence of immunoglobulin G aCL antibody is higher in patients with AS than in the normal population [8].

Another speculative pathophysiology of INCPH is venopathy. Obliterative portal venopathy is a characteristic morphologic feature in INCPH, leading to an increased intra-hepatic resistance. AS has been reported to be associated with accelerated atherosclerosis including coronary artery disease [9], so speculatively has the potential to initiate the obliterative portal venopathy.

It usually presents with symptoms or signs of gastroesophageal varices, ascites, encephalopathy, splenomegaly, or hyperplenism. Moreover, the liver function is usually preserved. In over $75 \%$ of INCPH patients, varices were discovered at the initial endoscopy. The 1-year probability of developing small and large varices was $10 \%$ and $13 \%$ respectively [2]. Splenomegaly was presented in the majority of Japanese and Western patients, whereas only a minority (14\%) was presented with splenomegaly in a large Indian study [6]. Splenomegaly and varices were not found in our patient.

INCPHis a clinical diagnosis, and no laboratory test has been discovered to confirm INCPH. This patient had a clinical sign of portal hypertension, such as perisplenic varices, but no history of chronic liver disease or any other conditions that may cause cirrhosis. Doppler ultrasound that shows patent portal and hepatic veins, as well as a liver biopsy that shows no cirrhosis, can lead to the diagnosis of INCPH. Although splenomegaly and varices were not found in the initial workup, they should be thoroughly evaluated in the follow-up.

A long-term prognosis remains unclear. INCPH has been considered as a benign disorder; however, over the past few years, there have been reports showing INCPH patients with poor long-term outcome, developing severe liver decompensation, multiorgan failure, and even death. A recent study reported poor survival, showing overall 10 -year survival rate to be $56 \%[10]$.

INCPH is a frequently unrecognized condition, and in many instances, patients are misdiagnosed as LC. The prognosis of patients with INCPH is generally better than that of those with cirrhosis; however, a small subgroup, such as INCPH associated with immunological diseases, showed a poor prognosis [2]. Therefore, it is important for clinicians to improve the understanding of its complex pathophysiology. To improve the management of $\mathrm{INCPH}$, promoting the awareness of INCPH remains to be the most important factor.

\section{CONFLICT OF INTEREST}

No potential conflict of interest relevant to this article was reported.

\section{ORCID}

Sukki Park, https://orcid.org/0000-0002-2387-9359

Ji Hyun Lee, https://orcid.org/0000-0002-9237-9083

\section{REFERENCES}

1. Schouten JN, Verheij J, Seijo S. Idiopathic non-cirrhotic portal hypertension: a review. Orphanet J Rare Dis 2015;10:67.

2. Siramolpiwat S, Seijo S, Miquel R, Berzigotti A, Garcia-Criado A, Darnell A, et al. Idiopathic portal hypertension: natural history and long-term outcome. Hepatology 2014;59:227685.

3. Hillaire S, Bonte E, Denninger MH, Casadevall N, Cadranel JF, Lebrec D, et al. Idiopathic non-cirrhotic intrahepatic portal hypertension in the West: a re-evaluation in 28 patients. Gut 2002;51:275-80.

4. Cazals-Hatem D, Hillaire S, Rudler M, Plessier A, Paradis $\mathrm{V}$, Condat B, et al. Obliterative portal venopathy: portal hypertension is not always present at diagnosis. J Hepatol 2011; 54:455-61.

5. Vaiphei K, Bhatia A, Sinha SK. Liver pathology in collagen 
vascular disorders highlighting the vascular changes within portal tracts. Indian J Pathol Microbiol 2011;54:25-31.

6. Schouten JN, Garcia-Pagan JC, Valla DC, Janssen HL. Idiopathic noncirrhotic portal hypertension. Hepatology 2011;54: 1071-81.

7. Ziol M, Poirel H, Kountchou GN, Boyer O, Mohand D, Mouthon L, et al. Intrasinusoidal cytotoxic CD8 + T cells in nodular regenerative hyperplasia of the liver. Hum Pathol 2004; 35:1241-51.

8. Juanola X, Mateo L, Domenech P, Bas J, Contreras N, Nolla JM, et al. Prevalence of antiphospholipid antibodies in pa- tients with ankylosing spondylitis. J Rheumatol 1995;22: 1891-3.

9. Ungprasert P, Srivali N, Kittanamongkolchai W. Risk of coronary artery disease in patients with ankylosing spondylitis: a systematic review and meta-analysis. Ann Transl Med 2015; 3:51.

10. Schouten JN, Nevens F, Hansen B, Laleman W, van den Born $\mathrm{M}$, Komuta $\mathrm{M}$, et al. Idiopathic noncirrhotic portal hypertension is associated with poor survival: results of a long-term cohort study. Aliment Pharmacol Ther 2012;35:1424-33. 